\title{
Mathematische Auffälligkeiten bei Rechenstörungen
}

\author{
Beobachtbare mathematische Auffälligkeiten bei Rechenstörungen als \\ Grundlage für diagnostische Maßnahmen
}

Nadine Hofbauer ${ }^{1}$

https://doi.org/10.53349/resource.2021.i16.a991

\section{Zusammenfassung}

Mathematische Auffälligkeiten erweisen sich während der Grundschulzeit als vielseitig und gleichermaßen als hilfreich, um Rechenstörungen frühzeitig erkennen zu können und somit die Grundlage für eine umfassende Diagnose sowie eine angemessene Förderung von betroffenen Kindern zu schaffen. Sowohl im privaten als auch im später beruflichen Bereich sorgen mathematische Lernschwierigkeiten für beeinträchtigende Herausforderungen, wobei ein frühzeitiges Erkennen die negativen Auswirkungen von Rechenstörungen minimieren kann. Der vorliegende Beitrag erläutert mathematische Auffälligkeiten bei Rechenstörungen, welche sich im schulischen Kontext zeigen. Insbesondere für Lehrpersonen werden häufig auftretende Erscheinungsformen sowie Symptome von Rechenstörungen beschrieben, welche die Beobachtungen mathematischer Lernschwierigkeiten ermöglichen und somit den Ausgangspunkt für umfassende diagnostische Maßnahmen darstellen.

\author{
Keywords: \\ Rechenstörung \\ Mathematische Auffälligkeiten \\ Numerisch-rechnerische Fertigkeiten \\ Symptome bei Rechenstörungen \\ Frühzeitiges Erkennen
}

\section{Einleitung}

Bereits im Kindergartenalter und besonders im Laufe der Grundschulzeit zeigen sich mathematische Auffälligkeiten, welche auf Rechenstörungen hinweisen können. Umfassende Lernschwierigkeiten im mathematischen Bereich sollen frühzeitig erkannt werden, um eine angemessene Förderung für betroffene Kinder vor oder während der Grundschulzeit anbieten zu können (vgl. Schipper, 2005, S. 55). Ausgeprägte Rechenfähigkeiten spiegeln sich im Erwachsenenalter sowohl im beruflichen Kontext als auch im alltäglichen Handeln wider. Sei es das Kopfrechnen während des Einkaufens, das richtige Schätzen der Größe einer Parklücke oder auch die Ausübung naturwissenschaftlicher Tätigkeiten: all diese Aktivitäten setzen umfassende Rechenfähigkeiten voraus. Da Rechnen eine vielfältige neurokognitive Herausforderung ist, lässt sich erschließen, dass sich große Herausforderungen für Menschen mit Rechenschwierigkeiten oder Rechenstörungen ergeben, die unmittelbaren Einfluss auf das Alltagsleben und die Berufswahl haben. Menschen mit Rechenschwierigkeiten oder Rechenstörungen gelingt das kompetente Arbeiten und Verarbeiten mit und von Zahlen kaum, wobei die Ursachen innerhalb von Teilkomponenten des Rechnens liegen können (vgl. Landerl, Vogel \& Kaufmann, 2017, S. 16). Um die Vielzahl an mathematischen Auffälligkeiten bei Rechenstörungen näher erläutern zu können, wird anfangs der Begriff der Rechenstörung näher bezeichnet.

\footnotetext{
${ }^{1}$ Pädagogische Hochschule Steiermark, Hasnerplatz 12, 8010 Graz. E-Mail: nadine.hofbauer@phst.at
} 


\section{Rechenstörung}

Der World Health Organization (WHO) zufolge wird die Rechenstörung nach der International Statistical Classification of Diseases and Related Health Problems (ICD-10) dem Bereich der umschriebenen Entwicklungsstörungen schulischer Fertigkeiten zugeordnet und somit den psychischen Störungen und den Verhaltensstörungen. Unter dem Punkt F81.2 der 10. Revision der „International Statistical Classification of Diseases and Related Health Problems" (ICD-10) wird der Rechenstörung die Beeinträchtigung von Rechenfertigkeiten zugeschrieben. Es finden sich Schwächen in der Addition, der Subtraktion, der Multiplikation sowie der Division. Die World Health Organization beschreibt eine allgemein minimierte Intelligenz, eine unangebrachte Beschulung oder höhere mathematische Fertigkeiten als weniger relevant (vgl. DIMDI, 2021). In der vierten Auflage des „Diagnostischen und statistischen Manuals für psychische Störungen“ (DSM-IV) wird die Rechenstörung hingegen als herabgesetzte mathematische Befähigung bezeichnet und nicht als Beeinträchtigung der Rechenfertigkeiten, wie das laut WHO der Fall ist. Übereinstimmend legen allerdings sowohl das Klassifikationssystem ICD-10 als auch das System DSM-IV drei „Kriterien für die Minderleistung bei Lernstörungen dar (d. Verf.): Die Leistung muss deutlich unter dem aufgrund (1) des Alters, (2) der allgemeinen Intelligenz und (3) der Beschulung zu erwartendem Niveau liegen“ (Mähler \& Grube, 2012, S. 606f). Moser Opitz verweist in der Definition von Rechenstörungen auf die WHO und streicht hervor, dass Rechenstörungen zu den Teilleistungsstörungen zählen und bei durchschnittlicher Intelligenz und Schulleistung auftreten (vgl. Moser Opitz, 2013, S. 17). Ergänzend charakterisieren Landerl, Vogel und Kaufmann den Begriff der Dyskalkulie anhand der Entwicklungsbeeinträchtigung in den Bereichen des Rechnens und der Verarbeitung von Zahlen, welche hinsichtlich der intellektuellen Leistungsfähigkeit faktisch nicht zu erwarten wäre (vgl. 2017, S. 223). Weitere Differenzierungen zeigen Jacobs und Petermann (2005) auf, wobei unterschieden wird zwischen Kindern mit mathematischen Lernstörungen und durchschnittlicher Intelligenz, Kindern mit eher rechenschwacher Leistung und geringerer Intelligenz und Kindern mit unterdurchschnittlicher Schulleistung sowie unterdurchschnittlicher Intelligenz, welche als lernbehindert gelten und an einem besonderen Unterricht teilnehmen. Kinder mit unterdurchschnittlicher Schulleistung sowie Intelligenz zeigen keine konkrete Rechenstörung, da sich die mathematischen Defizite aus einer allgemeinen Lernbehinderung ableiten (vgl. S. 72).

Rechenschwierigkeiten können somit in unterschiedlichen Formen auftreten und müssen dementsprechend vielseitig untersucht werden, um eine angemessene Förderung anbieten zu können. Für die Diagnose einer Rechenstörung soll eine weitreichende diagnostische Untersuchung umgesetzt werden, welche einerseits domänenspezifische Fähigkeiten, somit numerisch-rechnerische Fähigkeiten, andererseits domänenübergreifende Fähigkeiten, demzufolge intellektuelles Niveau, Sprache, visuell-räumliche Fähigkeiten, emotionale Gegebenheiten und Merkfähigkeit, überprüft. Die Diagnose einer Dyskalkulie muss von Personen mit umfassenden Kenntnissen hinsichtlich neurokognitiver Prozesse in den Bereichen der Zahlenverarbeitung sowie des Rechnens und fundierten Kompetenzen in der kinderpsychologischen Diagnostik durchgeführt werden (vgl. Landerl, Vogel \& Kaufmann, 2017, S. 178f).

Mehrere Quellen verweisen auf die Notwendigkeit einer Überprüfung der Intelligenz betroffener Kinder, um eine Rechenstörung diagnostizieren zu können. Das zusätzliche Heranziehen eines Intelligenztests als diagnostisches Mittel ist relevant, um sicherzustellen, dass sich die Rechenstörung nicht von einer generellen Intelligenzminderung herleitet (vgl. Mähler \& Grube, 2012, S. 607ff). Der Aspekt der Intelligenz wird somit in der Diagnose einer Rechenstörung häufig einbezogen, allerdings wird auch Kritik laut. Infrage gestellt wird, ob sich ein Unterschied hinsichtlich der Lernschwierigkeiten während des Erwerbs mathematischer Fähigkeiten zwischen Kindern mit hoher und niedriger Intelligenz zeigt. Besonders Kinder mit einem Intelligenzquotienten im unteren Normalbereich sind gefährdet, die Verwehrung einer Diagnose der Rechenstörung zu erfahren und demnach trotz mathematischer Schwierigkeiten weniger Unterstützung zu erhalten. Um diese Problematik zu lösen, kann unter anderem der Lernfähigkeit von Kindern auf den Grund gegangen werden. Das Kriterium der Lernfähigkeit untersucht den Lernfortschritt bei gutem Unterricht, wobei die Qualität des Unterrichts anhand des Lernfortschritts aller Schüler*innen einer Klasse gemessen wird. Neben dem Lernfortschritt wird auch die Leistung überprüft und mit der Leistung der weiteren Schüler*innen der Klasse verglichen. Die Zugänge sowie Perspektiven hinsichtlich der Diagnose von Rechenstörungen sind vielseitig und werden anhand neuer Erkenntnisse stets weiterentwickelt (vgl. Moser Opitz, 2013, S. 18ff).

Nicht nur die Definitionen und einzelnen Faktoren von Rechenstörungen können voneinander abweichen, auch die Begriffe zur Beschreibung von Schwierigkeiten des mathematischen Lernens sind breit gefächert: Dyskalkulie, Rechenstörung, Rechenschwäche oder mathematische Schulleistungsschwäche (vgl. Moser Opitz, 2013, S. 15). 
Da sich keine eindeutige Konkretisierung eines Begriffs zur Beschreibung mathematischer Rechenschwierigkeiten erkennen lässt, wird der Begriff der Rechenstörung nach ICD-10 verwendet.

\section{Mathematische Auffälligkeiten bei Rechenstörungen}

Zahlenfertigkeiten sowie Rechenfertigkeiten können erworben werden, wenn visuell-räumliche, aufmerksamkeitsbezogene und gedächtnisbezogene Verarbeitungsprozesse sowie ein ausgeprägtes Verständnis für Sprache grundlegend vorhanden sind. Diese kognitiven Fähigkeiten legen den Grundstein mathematischen Lernens. Bereits im Kindergartenalter wird festgestellt, dass Kinder, welche Schwierigkeiten im Bereich des Erwerbs der Zahlen- und Rechenfertigkeiten vorweisen, oftmals die Beschäftigung mit Memory, Puzzeln oder Bausteinen meiden, da ihnen diese Aufgaben schwerer fallen als anderen Kindern derselben Altersgruppe. Definierte basisnumerische Fähigkeiten in der Mathematik werden in Form von mengenbezogenem und zahlenbezogenem Vorwissen bereits im Kindergartenalter entwickelt und können mithilfe präventiver Maßnahmen unterstützend als Vorbeugung von Rechenschwächen gestärkt werden (vgl. Jacobs \& Petermann, 2012, S. 11f). Innerhalb der Grundschulen sind rund fünf Prozent der Schüler*innen von einer mathematischen Lernstörung betroffen, wobei eine fehlende Behandlung der Lernstörung zur Aufrechterhaltung der Rechenstörung im Erwachsenenalter führt (vgl. Kaufmann \& von Aster, 2012, S. 767).

Auffällig bei Kindern mit diagnostizierter Rechenstörung ist, dass oftmals die mathematischen Leistungen im Zahlenraum bis 100, aber auch im Zahlenraum bis 20 am Ende der zweiten Schulstufe schwer zu bewältigen sind. Kinder mit Rechenstörung verwenden am Ende der vierten Schulstufe zum Rechnen die Finger und aufwendige Zählstrategien. Erstaunlich ist ebenso, dass jene Kinder kürzlich gerechnete Aufgaben nicht wiedererkennen, diese Aufgaben somit neu berechnen und sich Zwischenergebnisse schwer merken. Auch die Überschreitung sowie die Unterschreitung des Zehners stellt eine große Herausforderung für rechenschwache Kinder dar. Die erkennbare Folge dieser umständlichen Problemlösungen ist, dass Kinder mit Rechenstörung die zeitlichen Erwartungen hinsichtlich mathematischer Operationen nicht erfüllen können. Während des Lernprozesses sind allerdings bei vielen Kindern Auffälligkeiten und Fehler zu beobachten. Daher ist es wichtig, die Fehler rechenschwacher Kinder von typischen Fehlern des überwiegenden Teils von Lernenden zu unterscheiden. Im Erwerb von Rechenfertigkeiten stehen die Häufigkeit, die qualitative Vielfalt sowie die Kontinuität von Fehlern im Mittelpunkt (vgl. Jacobs \& Petermann, 2012, S. 12). Übereinstimmend geben Spiegel und Selter (2007) an, dass mathematische Lernschwierigkeiten bei Kindern auf Rechenstörungen hinweisen, wenn die Schwierigkeiten häufig auftreten, die Lernschwierigkeiten dauerhaft bestehen und ebenso überfordernd sind. Entscheidend sind somit Faktoren wie die Kontinuität und die Dauer des fehlerhaften Rechnens. Besonders Lehrpersonen sollen die Unterschiede zwischen Kindern mit Rechenschwierigkeiten und Kindern, die für das Verstehen mathematischer Inhalte lediglich in wenigen Fällen mehr Zeit und Mittel benötigen, erkennen (vgl. S. 87).

Nach Kaufmann und von Aster finden sich mehrere numerisch-rechnerische Fertigkeiten, die überwiegend bei Kindern mit Rechenstörung für Verständnisprobleme sorgen:

- „Erlernen, Abrufen und Anwenden von numerisch-rechnerischem Wissen (inklusive Zahlenraumvorstellung, arithmetisches Fakten- und Prozedurenwissen)

- falsches/inadäquates Anwenden von Rechenstrategien

- Schwierigkeiten bezüglich der Generalisierung der gelernten Inhalte

- $\quad$ kein oder sehr eingeschränkter Wissenstransfer (das heißt, gelernte Inhalte werden nicht automatisch - nicht ohne Unterstützung - auf andere Aufgaben und/oder Problembereiche übertragen).“ (Kaufmann \& von Aster, 2012, S. 771)

Die mangelhaften numerisch-rechnerischen Fertigkeiten finden sich im Grundschulbereich als Symptome einer Rechenstörung wieder. Kindern mit Rechenstörung fehlt das Verständnis hinsichtlich der Rechenoperationen und der Mengenbegriff gestaltet sich als nicht gesichert. Auch Zählen sowie Merken der Zahlwortfolge fällt Kindern mit Rechenstörung schwer und Faktenwissen wird kaum gespeichert. Ebenso bereiten das dezimale Stellenwertsystem und der Wechsel der Darstellungsart von Zahlen Schwierigkeiten (vgl. Simon, 2008, S. 223). In der Erläuterung von Jacobs und Petermann werden Leitsymptome definiert, welche die Kategorie der Verständnisschwierigkeiten der Rechenoperationen inkludiert. Fehlendes Mengenwissen, Zählfehler, Transkodierungsfehler, Rechenfehler sowie Schwierigkeiten mit dem Stellenwertverständnis werden ebenfalls in den Leitsymptomen genannt (vgl. Jacobs \& Petermann, 2012, S. 12ff; Simon, 2008, S. 223). 
Die beschriebenen Leitsymptome überschneiden sich größtenteils mit den näher definierten Symptomen, die Wilhelm Schipper als essentiell für das Erkennen von Rechenstörungen anführt (vgl. Schipper, 2005, S. 29ff). Auch Nicksteit legt für das Deutsche Zentrum für Lehrerbildung Mathematik dieselben Symptome dar, die Schipper als ausschlaggebend für das Erkennen von Rechenstörungen bezeichnet (vgl. Nicksteit, 2010, S. 1ff).

\subsection{Zählendes Rechnen}

Zählendes Rechnen wird von Kindern im Vorschulalter und zu Beginn des ersten Schuljahres angewandt, um der Grundvorstellung der Addition nachzukommen. Ein Beispiel hierfür ist es, bei der Anweisung, drei und vier zusammenzurechnen, zuerst drei Plättchen zu legen, danach vier hinzugefügt und schlussendlich alle Plättchen zu zählen, um die Summe zu entdecken (vgl. Schipper, 2005, S. 29). Das Lösen von Rechenaufgaben wird in den Anfängen der Schullaufbahn zählend mit Material oder mit den Fingern erleichtert, wobei Kinder mit Rechenschwächen oder Rechenstörung in ihrer weiteren Entwicklung kaum Strategien nutzen und das zählende Rechnen als Lösungsweg bis in die zweite Schulstufe der Primarstufe verwenden (vgl. Nicksteit, 2010, S. 1). Zu Beginn der Schulzeit gilt das zählende Rechnen als notwendige Voraussetzung, um Schwierigkeiten beim Mathematiklernen zu verhindern. Nach Schipper wird das zählende Rechnen erst ab dem dritten Schuljahr der Primarstufe als problematisch angesehen, da hier bereits weitere Strategien angewandt werden sollen. Das zählende Rechnen erweist sich somit als Merkmal für mathematische Lernschwierigkeiten (vgl. Schipper, 2005, S. 30). Zählendes Rechnen führt oftmals zum Verrechnen um eines, beispielsweise wird bei der Rechnung $8+6$ bei acht zu zählen begonnen, wobei fälschlicherweise 13 das Ergebnis der Rechnung darstellt (vgl. Jacobs \& Petermann, 2012, S. 12ff). Zeitlich näher definiert ist das zählende Rechnen in der ersten Hälfte des zweiten Schuljahres im Zahlenraum bis 100 erkennbar, da die Rechengeschwindigkeit bei Kindern mit Schwierigkeiten deutlich langsamer als bei den Mitschüler*innen ausfällt. Im Gegensatz zum Rechnen im Zahlenraum bis 100 funktioniert das zählende Rechnen im Zahlenraum bis 20 in einem unauffälligen Tempo und daher ist nicht erkennbar, ob schnell gezählt wird oder sinnvolle Strategien angewandt werden. Der Zahlenraum bis 100 verlangsamt die Ergebnisfindung zählend rechnender Kinder und offenbart somit Rechenschwierigkeiten (vgl. Schipper, 2005, S. 30). Nicksteit unterstützt diesen Zugang und meint ebenso, dass zählend rechnende Kinder rasch zu einem Ergebnis finden und ihre Lösungsstrategie daher oftmals erst am Ende des ersten oder sogar des zweiten Schuljahres auffällt. Ergänzend wird angemerkt, dass zählend rechnende Kinder einige kreative Ideen vorweisen, um das zählende Rechnen zu verbergen. Möglichkeiten dafür sind, mit den Fingern auf eine Oberfläche zu tippen oder den Kopf während des Zählens zu nicken (vgl. 2010, S. 1f). Auch Schipper weist darauf hin, dass die Möglichkeit besteht, zählendes Rechnen zu beobachtet, wobei Kinder häufig ihre Hände unter dem Tisch oder hinter dem Rücken verstecken oder Gegenstände im Raum einbeziehen und während des Zählprozesses leichte Kopfbewegungen ausführen. Wie bereits durch Jacobs und Petermann erwähnt, weisen im Zahlenraum bis 20 Ergebnisse, die um eines mehr oder eines weniger ergeben, als das korrekte Ergebnis wäre, auf Schwierigkeiten beim Rechnen hin. Im Zahlenraum bis 100 handelt es sich um zehn mehr oder um zehn weniger als die Ergebniszahl. Schipper gibt allerdings zu bedenken, dass ein einzelner Hinweis unzureichend ist, um zählendes Rechnen zu erkennen, da die Merkmale zählenden Rechnens über längere Zeit bei unterschiedlichen Aufgabenstellungen wahrgenommen werden müssen, um das verfestigte zählende Rechnen sicherzustellen (vgl. 2005, S. 31).

\section{Sechs Faktoren zählenden Rechnens}

Die folgenden sechs konkreten Faktoren geben Aufschluss darüber, ob die Lösungsstrategie des zählenden Rechnens angewandt wird:

1. Die Zerlegung der Zahlen bis zehn stellt den ersten Hinweis dar. Das Ende des ersten Schuljahres markiert jenen Zeitpunkt, an dem Kinder die Zahlenzerlegung bis zehn beherrschen sollen, da sie die Voraussetzung des schrittweisen Rechnens ist. Zählend rechnenden Kindern unterlaufen in der Umsetzung der Zahlenzerlegung häufig Fehler oder die Geschwindigkeit des Rechnens gestaltet sich als auffällig langsam. Ein zählend rechnendes Kind schafft es, von acht auf zehn zu ergänzen, allerdings benötigt es auffällig mehr Zeit, um von zwei auf zehn zu ergänzen (vgl. Schipper, 2005, S. 32ff).

2. Ein weiterer Faktor umfasst die geringe Anzahl automatisierter Rechenaufgaben. Das erste Schuljahr der Primarstufe endet mit dem Wissen über sämtliche Additions- und Subtraktionsrechnungen im Zahlenraum bis 10 
sowie dem Wissen über die Verdoppelungen und Halbierungen im Zahlenraum bis 20. Zählend rechnende Kinder können nur auf wenige automatisierte Aufgaben zurückgreifen und stellen aufgrund des enormen Zählaufwandes keine Verbindung zwischen der Rechenaufgabe und dem Ergebnis her. Neben dem großen Aufwand, eine Rechenaufgabe zählend zu lösen, ist auch die Fehlerquote der Ergebnisse auffällig hoch, wobei die hohe Fehlerquote wiederum die Herstellung der Verbindung zwischen Aufgabe und Ergebnis unterbindet (vgl. Schipper, 2005, S. 32ff).

3. Herausfordernd gestaltet sich auch der dritte Faktor des zählenden Rechnens. Operative Strategien können von zählend rechnenden Kindern angewandt werden, allerdings geschieht das selten. Können operative Strategien zur Lösungsfindung verwendet werden, wählen zählend rechnende Kinder dennoch das Zählen, da ihnen diese Methode vertraut ist (vgl. Schipper, 2005, S. 32ff).

4. Der vierte Faktor behandelt die Thematik des Ziffernrechnens, da zählend rechnende Kinder bei Aufgaben mit hohen Zahlen die Strategie entwickeln, nicht nach Stellenwert, sondern nach Ziffern zu rechnen. Aufgrund des mangelnden Stellenwertverständnisses treten beim Ziffernrechnen verstärkt Stellenwertfehler auf, die besonders bei Über- und Unterschreitungen auffallen (vgl. Schipper, 2005, S. 32ff). Jacobs und Petermann sehen im fehlenden Verständnis des Stellenwertsystems ebenso das Potenzial, Hinweise einer Rechenstörung zu erkennen. Beispielsweise werden Ziffern willkürlich zusammengerechnet und Zahlenübergänge kaum beachtet (vgl. 2012, S. 12ff).

5. Ein weiterer Faktor des zählenden Rechnens lässt erkennen, dass fehlendes Verständnis durch Rechenregeln kompensiert wird. Hier wenden zählend rechnende Kinder oftmals die gleiche Rechenregel an und erfahren dank dieser Regel teilweise Erfolg. Bei genauerer Betrachtung stechen allerdings Fehler hervor, die auf das Unverständnis der Rechenregeln hindeuten (vgl. Schipper, 2005, S. 32ff). So werden unteranderem Analogieaufgaben kaum verstanden und falsch transferiert. Die Aufgabe $5+4$ wird zu $4+5$, doch auch aus $5-4$ wird $4-5$, wobei eine Rechenregel genutzt und falsch verwendet wurde (vgl. Jacobs \& Petermann, 2012, S. 12ff).

6. Ergänzend erläutert der sechste Faktor, dass das Erkennen und Nutzen von Strukturen oftmals bei zählend rechnenden Kindern fehlend ist, da jene Kinder didaktische Hilfsmaterialien zählend verwenden und diese Kinder keine anwendbare Struktur zur Unterstützung einer rechnerischen Lösungsfindung nutzen (vgl. Schipper, 2005, S. 32ff). Ebenso bezeichnet Nicksteit das Auswendiglernen von Rechenoperationen als Merkmal, um Lernschwierigkeiten im mathematischen Bereich zu erkennen. Mit dem fehlenden Verständnis einer mathematischen Handlung ist auch die Entwicklung von Operationsvorstellung schwierig. Beispielsweise interpretiert ein Kind mit mathematischen Lernschwierigkeiten, dass das Additionssymbol bedeutet, weiterzuzählen und setzt diese Strategie auch bei der Lösung von Ergänzungsaufgaben um. Aus der Rechnung 3 $+\ldots=4$ entsteht aufgrund des Weiterzählens das Ergebnis 7, anstatt der zu ergänzenden 1. Mathematische Operationen werden somit nicht verstanden, sondern auswendig gelernt und umgesetzt (vgl. 2010, S. 3). Häufig werden falsche Ergebnisse von Kindern mit Rechenschwierigkeiten als Folge der Anwendung einer falschen Rechenregel interpretiert. Ist das der Fall, fehlt ein umfassendes Operationsverständnis (vgl. Spiegel \& Selter, 2007, S. 90). Zählendes Rechnen und besonders die Verfestigung dieser Vorgehensweise kann arithmetische Lernprozesse fortwährend negativ beeinflussen, weshalb ein frühzeitiges Erkennen sinnhaft ist (vgl. Schulz, 2014, S. 91ff).

\subsection{Links-/ Rechts-Problematik}

Nicht nur das zählende Rechnen deutet auf eine Rechenstörung hin, auch die Unterscheidung zwischen den beiden Richtungsangaben links und rechts fällt besonders Kindern mit Rechenstörung schwer. Dieser Aspekt kann ein wichtiger Hinweis für das Erkennen von Rechenstörungen sein. Schwierigkeiten mit dem Umgang von links und rechts sind eine große Herausforderung, da didaktisches Material, wie beispielsweise der Zahlenstrahl, oftmals mit den Richtungen links sowie rechts arbeitet (vgl. Spiegel \& Selter, 2007, S. 88). Auch Schipper bezeichnet die Problematik von vielen Kindern mit Rechenstörung in der Unterscheidung zwischen links und rechts als besonders auffällig. Die beiden Richtungen werden von betroffenen Kindern weder an der eigenen Person, noch am Gegenüber sicher erkannt. Schipper betont, dass mathematisches Arbeitsmaterial mithilfe der Richtungen veranschaulichend funktioniert und die Kenntnis über die Richtungen links und rechts entscheidend für sinnhaftes Mathematiklernen mithilfe didaktischen Materials ist. Zu überprüfende Hinweise für Rechenstörungen können die spiegelbildliche Schreibweise von Ziffern oder auch Zahlendreher sein, wobei etwa statt der Zahl 32 die Zahl 23 geschrieben wird. Ein weiteres Merkmal kann die Reihenfolge des Zahlenschreibens sein, wenn also bei der Zahl 32 zuerst die zwei und danach die drei geschrieben wird. Da die Rechenergebnisse infolge der Links-/ Rechts-Problematik fehlerhaft sind, ist es wichtig, den betroffenen Kindern zu veranschaulichen, wie diese Fehler zustande kommen. Ebenso soll ein Verständnis für die korrekte 
Vorgehensweise geschaffen werden (vgl. 2005, S. 47f). Im Bereich der Links-/ Rechts-Problematik merken ebenso Jacobs und Petermann an, dass sowohl ein Verdrehen als auch ein Verwechseln von Ziffern bei der Verschriftlichung der Zahlen bei Kindern mit Rechenstörung häufig vorzufinden sind (vgl. 2012, S. 12ff).

\subsection{Intermodalitätsprobleme}

Mathematisch auffällig ist, dass Kinder mit Rechenstörung Schwierigkeiten in der Vernetzung zwischen der enaktiven, ikonischen und symbolischen mathematischen Darstellung zeigen. Die enaktiv mathematische Ebene stellt Inhalte anhand einer konkreten Handlung dar, welche haptisch durchgeführt wird. Die ikonische Ebene arbeitet mit der Veranschaulichung durch Bilder oder Grafiken und die symbolische Darstellungsebene umfasst Zeichen, Symbole und die Sprache. Die Übersetzung von der einen Ebene auf die andere Ebene gestaltet sich für Kinder mit Rechenstörung als besonders fordernd. Wird beispielsweise innerhalb einer Aufgabenstellung verlangt, eine Rechengeschichte mithilfe von didaktischem Material darzustellen, soll von der symbolischen Ebene auf die enaktive Ebene übersetzt werden. Ein Exempel einer solchen Aufgabenstellung wäre, wenn ein Mädchen vier Puppen hat und noch zwei Puppen geschenkt bekommt, wobei die Frage nun lautet, wie viele Puppen das Mädchen insgesamt hat. Die Handlung der Rechengeschichte soll mithilfe von Legematerial dargestellt und die mentale Vorstellung der Addition soll geschult werden (vgl. Schipper, 2005, S. 49f). Wie bereits erwähnt, fällt der Wechsel zwischen den Ebenen besonders Kindern mit Rechenstörung schwer. Häufig verweilen betroffene Kinder auf der Ebene der Zahlsymbole, da sich ihnen die Sinnhaftigkeit des Übersetzens in eine andere Ebene nicht erschließt. Auch das als Unterstützung gedachte Material kann sich als unnötig erweisen, wenn Kinder mit mathematischen Lernschwierigkeiten den Zusammenhang zwischen der Rechenaufgabe und dem Material nicht erkennen (vgl. Spiegel \& Selter, 2007, S. 90). De Vries schließt sich der Meinung zur Relevanz der enaktiven, ikonischen und symbolischen Ebenen für ein sinnvolles mathematisches Lernen an. Besonders die enaktive Ebene, somit die Handlungsebene, soll Abläufe und Zusammenhänge erkennen und verstehen lassen. Mathematische Abläufe sollen mithilfe didaktischen Materials und dessen Anwendung verinnerlicht werden, um die Basis für weitere Abstraktionen zu schaffen und die ikonischen sowie die symbolischen Darstellungsformen einzubeziehen (vgl. 2018, S. 11ff).

\subsection{Einseitige Zahl- und Operationsvorstellungen}

Häufig kommt es neben Intermodalitätsproblemen auch zu einseitigen Zahl- und Operationsvorstellungen, da Kinder mit Rechenstörung Zahlen häufig als Ziffern definieren und weitere Darstellungsformen außer Acht lassen. Ein konkretes Beispiel für einseitige Zahl- und Operationsvorstellungen ist die bildnerische Darstellung der Aufgabe $2 \times 6$, wobei Kinder mit Rechenstörung die Zahlen häufig als Menge und nicht als Operation darstellen. Statt sechs gezeichneten Luftballons und nochmals sechs gezeichneten Luftballons, welche das richtige Ergebnis von zwölf Luftballons darstellen, werden zwei Luftballons, das Multiplikationszeichen und weitere sechs Luftballons gezeichnet. Die Zahl- und Operationsvorstellung der wiederholten Addition innerhalb der Multiplikation wird somit nicht dargestellt. Wie bereits hinsichtlich der Intermodalitätsprobleme erläutert, gelingt Kindern mit Rechenstörung der Wechsel zwischen der enaktiven, der ikonischen und der symbolischen Ebene nur schwer (vgl. Schipper, 2005, S. 53ff). Die Transferleistung ist entscheidend, um eine adäquate mathematische Grundvorstellung zu erreichen. Um Rechenoperationen zu verstehen, müssen die individuellen Erfahrungen sowie Vorstellungen der Schüler*innen mit den Sachzusammenhängen, den mathematischen Grundvorstellungen und den mathematischen Begriffen und Verfahren in Verbindung gebracht werden. Werden Rechenoperationen nicht sinnhaft verstanden, werden mathematische Aufgabenstellungen anhand auswendig gelernter Regeln gelöst und ein weiteres Verständnis verhindert (vgl. Leiß, 2003, S. 24).

Die Problematik eines fehlenden Operationsverständnisses erkennen auch Kaufmann und Wessolowski. Eine einfache Sachaufgabe kann sich ohne Operationsverständnis zu einer echten Herausforderung entwickeln und damit zu einer wichtigen Auffälligkeit zur Erkennung einer Rechenstörung werden (vgl. 2006, S. 14). Des Weiteren erklärt die Definition einer Zahl als Position innerhalb der Zahlwortreihe nochmals das einseitige Verständnis von Zahlen seitens der Kinder mit Rechenstörung. Hierbei wird die Zahl 8 als achte Perle an einer Kette verstanden und nicht als die Summe aller Perlen bis einschließlich der achten Perle. In diesem Fall wird vorrangig die Ordinalzahl bedacht und die Anzahl einer Zahl vernachlässigt (vgl. Kaufmann \& Wessolowski, 2006, S. 14). Fehlend ist somit das Kardinalzahlverständnis, welches das Wissen um Mengen betrifft und den Nachteil nach sich zieht, dass die Strategien der Mengenzerlegungen und der Zahlbeziehungen zur Verwendung während 
mathematischer Lösungsvorgänge nicht zur Verfügung stehen (vgl. Nicksteit, 2010, S. 2). Erkennbar ist ebenso, dass Kinder mit mathematischen Lernschwierigkeiten im Einschätzen von Mengen Probleme vorweisen, kaum Überschlagsrechnungen anwenden und unrealistische Rechenergebnisse selten erkennen (vgl. Jacobs \& Petermann, 2012, S. 12ff).

Sichtlich sind die mathematischen Auffälligkeiten bei Rechenstörungen besonders facettenreich. Die Formen, in welcher Rechenstörungen auftreten können, sind einerseits generalisierbar, andererseits in ihrer Erscheinung so individuell, wie das auch bei den unterschiedlichen Persönlichkeiten der Kinder der Fall ist. Um weitere diagnostische Maßnahmen zu treffen, sind eine starke Häufung der Auffälligkeiten und ein zeitlich dauerhaftes Vorkommen dieser Auffälligkeiten notwendig. Die Vielfalt an Auffälligkeiten erweist sich demnach als entscheidende Grundlage, um Rechenstörungen frühzeitig mithilfe diagnostischer Schritte zu definieren und eine angemessene Förderung für betroffene Kinder zu erlangen (vgl. Schipper, 2005, S. 55).

\section{Literatur}

De Vries, C. (2018). Mathematik im Förderschwerpunkt Geistige Entwicklung: Grundlagen und Übungsvorschläge für Diagnostik und Förderung im Rahmen eines erweiterten Mathematikverständnisses. (4. Aufl.). Modernes Lernen Dortmund.

Deutsches Institut für Medizinische Dokumentation und Information - DIMDI (2021, Juli 21). ICD-10-WHO Version 2019 - Kapitel V Psychische und Verhaltensstörungen (FOO-F99). https://www.dimdi.de/static/de/klassifikationen/icd/icd-10-who/kode-suche/htmlamt|2019/blockf80-f89.htm

Jacobs, C. \& Petermann, F. (2005). Diagnostik von Rechenstörungen - Kompendien Psychologische Diagnostik. Hogrefe Verlag GmbH \& Co. KG Göttingen.

Jacobs, C. \& Petermann, F. (2012). Diagnostik von Rechenstörungen. (2. Aufl.). Hogrefe Verlag GmbH \& Co. KG Göttingen.

Kaufmann, L. \& von Aster, M. (2012). Diagnostik und Intervention bei Rechenstörung. Deutsches Ärzteblatt Int 2012, 109(45), S. 767 - 78. https://www.aerzteblatt.de/pdf.asp?id=132184

Kaufmann, S. \& Wessolowski, S. (2006). Rechenstörungen. Diagnose und Förderbausteine. Kallmeyer Verlag Seelze.

Landerl, K. \& Vogel, S. \& Kaufmann, L. (2017). Dyskalkulie. (3. Aufl.). Ernst Reinhardt Verlag München Basel.

Leiß, D. (2003). Arbeitstechniken im Mathematikunterricht. Begriffsklärung, Beispiele und empirische Erhebungen. Zentrum für Lehrerbildung der Universität Kassel 2003, 144 S. - (Reihe Studium und Forschung; 4). http://nbn-resolving.org/urn:nbn:de:0111-opus-18129

Mähler, C. \& Grube, D. (2012). Lernstörungen. In W. Schneider \& U. Lindenberger (Hrsg.). Entwicklungspsychologie. (7. Aufl.). Beltz Verlag Weinheim Basel (S. 605-618).

Moser Opitz, E. (2013). Rechenschwäche/ Dyskalkulie. Theoretische Klärungen und empirische Studien an betroffenen Schülerinnen und Schülern. (2. Aufl.). Haupt Verlag Bern.

Nicksteit, J. (2010). Wie kann ich eine Rechenschwäche bei meinen Schülern erkennen? https://pikas.dzlm.de/pikasfiles/uploads/upload/Material/Haus 3 Umgang mit Rechenschwierigkeiten/IM/Informationstexte/H3 IM Wie kann ich eine Rechensch wache erkennen.pdf

Schipper, W. (2005). Lernschwierigkeiten erkennen - verständnisvolles Lernen fördern. IPN Universität Kiel Kiel. Schulz, A. (2014). Fachdidaktisches Wissen von Grundschullehrkräften: Diagnose und Förderung bei besonderen Problemen beim Rechnenlernen. Springer Fachmedien Wiesbaden.

Simon, H. (2008). Dyskalkulie - Kindern mit Rechenschwäche wirksam helfen. (2. Aufl.). Klett-Cotta Stuttgart.

Spiegel, H. \& Selter, C. (2007). Kinder und Mathematik. Was Erwachsene wissen sollten. Kallmeyer Verlag Seelze. 